\title{
Osteoinductive and anti-inflammatory effect of royal jelly on periodontal ligament cells
}

\author{
Manabu Yanagita, Yuko Kojima, Kenta Mori, Satoru Yamada, and Shinya Murakami \\ Department of Periodontology, Division of Oral Biology and Disease Control, Osaka University Graduate School of Dentistry, Osaka \\ 565-0871, Japan
}

(Received 17 June 2011; and accepted 30 June 2011)

\begin{abstract}
Royal jelly (RJ) has been reported to possess several physiological and pharmacological properties such as the ability to prevent osteoporosis in rats and anti-inflammatory effects. We hypothesized that RJ could have beneficial effects on the prevention or treatment of periodontal diseases, which are chronic inflammatory diseases caused by bacterial infection that result in resorption of the tooth-supporting bone. We assessed the effect of RJ on mineralization in mouse periodontal ligament cell clone 22 (MPDL22 cells), which are of an osteogenic and cementogenic lineage. The mRNA expression of osteopontin, osteocalcin and osterix, and mineralized nodule formation were significantly enhanced in RJ-treated MPDL22 cells. In addition, we investigated the effects of RJ on the production of inflammatory cytokines from MPDL22 cells stimulated with lipopolysaccharide (LPS) of Porphyromonas gingivalis, a periodontopathic bacterium. RJ suppressed LPSinduced interleukin- 6 and CXC chemokine ligand 10 production from MPDL22 cells. Furthermore, RJ suppressed the expression of CD54 in MPDL22 cells: CD54 is the adhesion molecule involved in the accumulation of leukocytes in periodontal lesions. These findings suggest that the osteoinductive and anti-inflammatory effects of RJ can provide benefits for the treatment and prevention of periodontal diseases.
\end{abstract}

Periodontal disease is a bacterial biofilm-induced chronic inflammatory disease characterized by the destruction of periodontal tissues including the periodontal ligament (PDL), cementum, gingiva and alveolar bone $(10,24)$. Among these, PDL, which is located between the alveolar bone and cementum of the tooth, plays crucial roles in the homeostasis and repair/regeneration of periodontal tissues. PDL has been revealed to possess multipotential mesenchymal stem cells that can differentiate into osteoblasts and cementoblasts which can create alveolar bone

Address correspondence to: Dr. Shinya Murakami, PhD, D.D.S., Department of Periodontology, Division of Oral Biology and Disease Control, Osaka University Graduate School of Dentistry, Yamadaoka 1-8, Suita, Osaka 565-0871, Japan

Tel: +81-6-6879-2930, Fax: +81-6-6879-2934

E-mail: ipshinya@dent.osaka-u.ac.jp and cementum (21), respectively, and to express bone-related markers $(2,18)$. Furthermore, several lines of evidence have shown that PDL cells produce inflammatory cytokines and chemokines, such as interleukin (IL)-6 and IL-8 in response to lipopolysaccharide (LPS) of Porphyromonas gingivalis (P. gingivalis), one of the causative black-pigmented Gram-negative anaerobes, responsible for the development of chronic inflammation in the periodontium (27). Thus, PDL cells are involved in the repair and maintenance of the periodontium, as well as in inflammation.

Royal jelly (RJ) is produced in the hypopharyngeal and mandibular glands of worker honeybees (Apis mellifera) and is a necessary food for the growth of the queen honeybee. RJ consists of proteins $(18 \%)$, sugars (15\%), lipids (3-6\%) and water (50-60\%). RJ also contains vitamins, amino acids and minerals (17). Interestingly, RJ has been shown to exhibit 
several biological activities, including anti-hypercholesterolemic (25), anti-tumor (3), anti-microbial (4), anti-allergic (20), anti-inflammatory properties (11), and vasodilative and hypotensive activities (22). Recent studies have shown that RJ has weak estrogenic effects and prevents osteoporosis in ovariectomized rats $(7,15)$. Like osteoporosis, periodontal diseases are typically accompanied by bone resorption. In fact, the positive association between osteoporosis and periodontitis has been suggested (13). We hypothesized that administration of RJ in the daily diet or local application of RJ to periodontal tissue might prevent periodontal disease.

In the present study, we investigated the effects of $\mathrm{RJ}$ on osteogenic mineralized nodule formation using mouse PDL (MPDL) cells and on the secretion of IL-6 as inflammatory cytokine and CXC chemokine ligand 10 (CXCL10), chemokine suggested to be involved in alveolar bone destruction in periodontal diseases lesion (9), from LPS-stimulated MPDL cells.

\section{MATERIALS AND METHODS}

Reagent. Dried powders of raw RJ originating from China were supplied by Yamada Apiculture Center, Inc. (Okayama, Japan). LPS of $P$. gingivalis was purchased from InvivoGen (San Diego, CA, USA).

Culture of $M P D L$. In a previous work, we established an MPDL clone cell line, MPDL22, isolated from the PDL tissue of the molar teeth extracted from 2.5-week-old BALB/c mice (26). MPDL22 cells were maintained in $\alpha$-MEM (Nikken, Kyoto, Japan) supplemented with $10 \%$ fetal calf serum (FCS; JRH Biosciences, Lenexa, KS, USA) and $100 \mathrm{ng} / \mathrm{mL}$ fibroblast growth factor-2 (FGF-2; Kaken, Kyoto, Japan) (26). When the cells reached confluence, we replaced the culture medium ( $\alpha$-MEM supplemented with $10 \%$ FCS and FGF-2) with the mineralization medium ( $\alpha$-MEM supplemented with $10 \%$ FCS, $10 \mathrm{mM} \beta$-glycerophosphate, and $50 \mathrm{mg} /$ $\mathrm{mL}$ ascorbic acid).

Proliferation assay. MPDL22 cells $\left(5 \times 10^{3}\right.$ cells/ well) were incubated in 96-well plates in $\alpha$-MEM containing 1\% FCS in the presence of RJ or FGF-2 $(100 \mathrm{ng} / \mathrm{mL})$ for $48 \mathrm{~h}$. Cell proliferation was measured using the nonradioactive colorimetric assay WST-1 assay (Roche Diagnostics GmbH, Penzberg, Germany) according to the manufacturer's instructions. The OD450/650 was measured after $2 \mathrm{~h}$ on a microplate reader (Bio-Rad, Hercules, CA).
Real-time PCR for osteopontin, osteocalcin and osterix $m R N A$. RNA samples were obtained from MPDL22 cells cultured in mineralization medium for 3, 5, and 7 days in the presence or absence of RJ. Total RNA extract $(0.4 \mathrm{mg})$ was reverse-transcribed using a High Capacity cDNA Reverse Transcriptase kit (Applied Biosystems, Foster City, CA, USA) to generate single-stranded cDNA. PCR reactions were carried out using the ABI 7300 Fast Real-Time PCR System (Applied Biosystems) with Power SYBR ${ }^{\mathrm{R}}$ Green PCR Master Mix (Applied Biosystems) according to the manufacturer's protocol. All reactions were run in triplicate. The primer sequences used for real-time PCR were as follows; osteopontin, (sense) 5'-TAC GAC CAT GAG ATT GGC AGT GA-3', (antisense) 5'-TAT AGG ATC TGG GTG CAG GCT GTA A-3'; osteocalcin, (sense) 5'-AGC AGC TTG GCC CAG ACC TA-3', (antisense) 5'-TAG CGC CGG AGT CTG TTC ACT AC-3'; osterix, (sense) 5'-CGC ATC TGA AAG CCC ACT TG-3', (antisense) 5'-CAG CTC GTC AGA GCG AGT GAA-3'.

Mineralization assay. Calcified nodules were stained by an alizarin red staining method. Cell layers were washed twice with phosphate-buffered saline (PBS) and then fixed in dehydrated ethanol. After fixation, the cell layers were stained with $1 \%$ alizarin red in $0.1 \% \mathrm{NH}_{4} \mathrm{OH}(\mathrm{pH} 6.3-6.5)$ for $5 \mathrm{~min}$. The culture dishes were then washed with water and observed, digitized and analyzed using WinRoof software (Mitani Corporation, Fukui, Japan).

Measurement of CXCL10 and IL-6 production. MPDL22 cells were seeded in 24-well culture dishes with $\alpha$-MEM supplemented with $10 \%$ FCS and $100 \mathrm{ng} / \mathrm{mL}$ FGF-2. When the cells reached $60-70 \%$ confluence, quiescent was induced by replacing the medium with serum-free $\alpha$-MEM for $24 \mathrm{~h}$, and then the medium was replaced with $\alpha$-MEM supplemented with $1 \%$ FCS with or without $P$. gingivalis LPS and RJ. The supernatants from the MPDL22 cultures were collected after $48 \mathrm{~h}$. The concentrations of CXCL10 and IL-6 in the supernatants were measured by ELISA (R\&D Systems, Minneapolis, MN, USA) according to the manufacturer's instructions. In some experiments, MPDL22 cells were cultured for $1,2,4$, or $6 \mathrm{~h}$ in the presence of RJ prior to LPS stimulation.

Flow cytometric acquisition. MPDL22 cells were seeded in 24-well culture dishes with $\alpha$-MEM supplemented with $10 \%$ FCS and $100 \mathrm{ng} / \mathrm{mL}$ FGF-2. 
When the cells reached $60-70 \%$ confluence, quiescent was induced by replacing the medium with serum-free $\alpha$-MEM for $24 \mathrm{~h}$, and then the medium was replaced with $\alpha$-MEM supplemented with $1 \%$ FCS with or without $P$. gingivalis LPS and RJ. After $48 \mathrm{~h}$, the cells were washed with PBS harvested by incubation with Cell Dissociation Solution (SigmaAldrich). Cells were washed with PBS and stained with phycoerythrin-conjugated rat anti-mouse CD54 antibody (eBiosciences, San Diego, CA, USA) for $30 \mathrm{~min}$. The cells were washed twice and data were acquired using FACSCalibur (BD Biosciences, San Jose, CA, USA). Analyses of viable cells were performed using CELLQuestTM software (BD Biosciences).

Statistical analysis. Results were analyzed for statistical significance using analysis of variance with the Bonferroni test. Differences were considered significant at $P$ values less than 0.05 . The mRNA expression levels were normalized against the expression levels of hypoxanthine phosphoribosyltransferase 1 (HPRT).

\section{RESULTS}

\section{Effect of RJ on MPDL22 proliferation}

To investigate the effect of RJ on the proliferation response of MPDL cells, cells were cultured for $48 \mathrm{~h}$ in a medium containing $1 \%$ FCS with or without RJ. As previous studies have shown that FGF-2 induced the proliferation of PDL cells (28), FGF-2 at a concentration of $100 \mathrm{ng} / \mathrm{mL}$, which was the most optimal concentration for MPDL proliferation in preliminary experiment, was used as a positive control of this assay. Exposure to $0.004-0.5 \mathrm{mg} / \mathrm{mL}$ RJ did not induce significant proliferative responses in MPDL22 cells (Fig. 1).

Effects of RJ on osteopontin, osteocalcin and osterix gene expression in MPDL22 cells

We examined the effects of RJ $(0.5 \mathrm{mg} / \mathrm{mL})$ on gene expression of extracellular matrices such as osteopontin and osteocalcin, and osteoblastic transcription factor, osterix (Fig. 2). RJ-treated-MPDL22 cells showed significantly enhanced mRNA expression of osteopontin (day 5), osteocalcin (day 3, 7) and osterix (day 3, 5, 7). Although RJ enhanced osterix gene expression at all points we investigated, there was the difference of gene expression pattern between osteopontin and osteocalcin. Whereas osteopontin expression was upregulated with peak at day 5 , osteocalcin had two peaks of mRNA expression

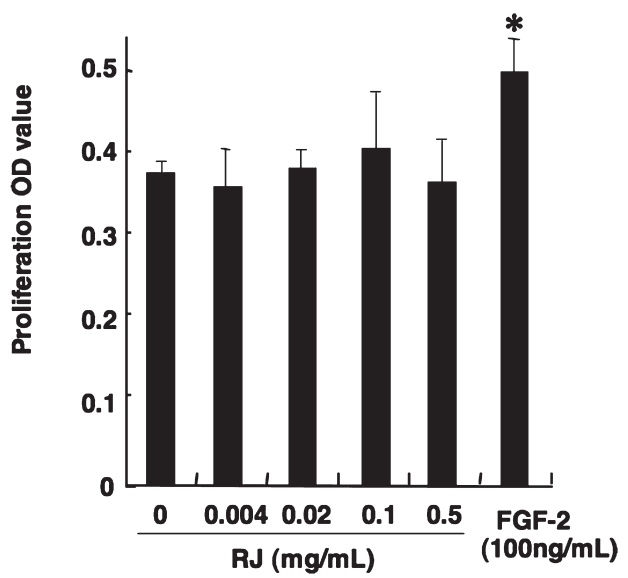

Fig. 1 Effect of RJ on proliferation of MPDL22 cells. MPDL22 cells were cultured with various doses of RJ or FGF-2 (100 ng/mL) for two days. RJ did not induce significant proliferative responses in MPDL22 cells. Values are the means $\pm \mathrm{SD}$ of four assays. ${ }^{*} P<0.05$ compared with medium only.

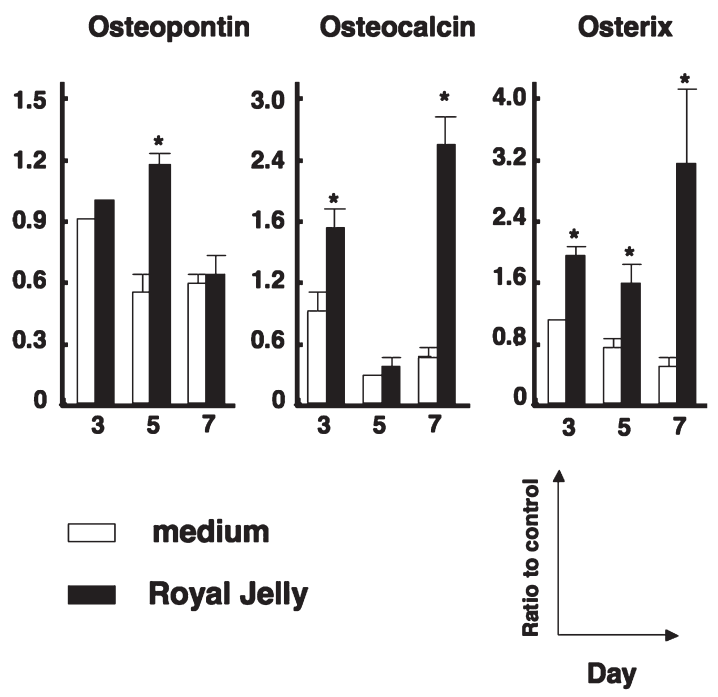

Fig. 2 Analysis of mRNA expression of osteopontin, osteocalcin and osterix in MPDL22 cells. RNA samples were obtained from MPDL22 cells at 3, 5 and 7 days after RJ treatment $(0.5 \mathrm{mg} / \mathrm{mL})$. The relative expression of each gene was normalized to the expression levels of HPRT, and the expression of osteopontin, osteocalcin and osterix in MPDL22 cells without $\mathrm{RJ}$ was taken as 1.0 . RJ significantly enhanced mRNA expression of osteopontin, osteocalcin and osteorix in MPDL22 cells. Values are the means \pm SD of three or four assays. ${ }^{*} P<0.05$ compared with non-treated cells.

at day 3 and day 7. The mechanism of suppression of osteocalcin expression at day 5 was unclear, however enhanced mRNA expression of osteocalcin at day 7 suggested emerging mature osteoblastic cells in the presence of RJ. 
Effects of RJ on mineralization in MPDL22 cells We cultured MPDL22 cells with or without RJ in mineralization medium and then examined mineralized nodule formation on day 12 . We exposed confluent MPDL22 cells to RJ at various concentrations. As shown in Fig. 3, RJ significantly enhanced alizarin red staining intensity, and in particular, addition of $0.1 \mathrm{mg} / \mathrm{mL}$ RJ was most effective.

\section{Effects of $R J$ on anti-inflammatory responses in MPDL22 cells}

To investigate the effects of RJ on the LPS-stimulated CXCL10 production, IL-6 secretion and CD54 expression, MPDL22 cells were pretreated with the indicated concentration of RJ for $1 \mathrm{~h}$ and stimulated with LPS for $48 \mathrm{~h}$. As shown in Fig. 4A, a dose-dependent suppressive effect of RJ on CXCL10 production by MPDL22 cells was observed. Although more than half of reduction of CXCL10 production in the prersence of RJ was shown, an approximately $30 \%$ reduction in IL-6 production was observed at each concentration of RJ investigated (Fig. 4B). The most effective dose of RJ for inhibition of IL- 6 production was $0.1 \mathrm{mg} / \mathrm{mL}$. CXCL10 and IL-6 inhibition was most efficient when RJ treatment occurred $6 \mathrm{~h}$ before LPS stimulation (Fig. 4C and D).

A previous study demonstrated that bacterial components induced a cell surface molecule CD54 in human PDL cells (12). As shown in Fig. 5A, MPDL22 cells without RJ and LPS stimulation expressed low level of CD54 (sample ID. 1), and treatment with only RJ had no effect on CD54 expression (sample ID. 2). MPDL22 cells with LPS strongly enhanced expression of CD54 (sample ID. 3). When we evaluated the expression of CD54 in MPDL22 cells pretreated with RJ $1 \mathrm{~h}$ prior to LPS stimulation, pretreatment with RJ reversed the LPS-induced upregulation of CD54 (sample ID. 4).

\section{DISCUSSION}

In the present study, we observed that RJ increased the formation of mineralized nodules in mouse PDL cells by enhancing osteoblastic differentiation. Additionally, RJ significantly suppressed LPS-induced inflammatory cytokine and chemokine production by MPDL22 cells.

Real-time PCR revealed that RJ enhanced the mRNA expression of osteopontin, osteocalcin, and osterix. Osteopontin is an indicator of bone turnover, and osteocalcin is involved in extracellular matrix mineralization. Osterix is an osteoblast-specific transcription factor that plays an important role in modulating bone formation and osteoblastic differentiation. Upregulation of the above-mentioned mRNAs by RJ correlated with the mineralization of MPDL22 cells. A previous study showed that RJ has estrogenic effects including the prevention of osteoporosis in ovariectomized rats $(7,15)$. Additionally, RJ has been reported to activate bone metabolism-related genes in the mouse osteoblast-like cell line, MC3T3-E1 (19). Because RJ comprises many components, the effects of RJ on promoting the expression of osteoblastic markers and mineralization in MPDL22 cells may be independent of its estrogenic activity. Isolation and characterization of

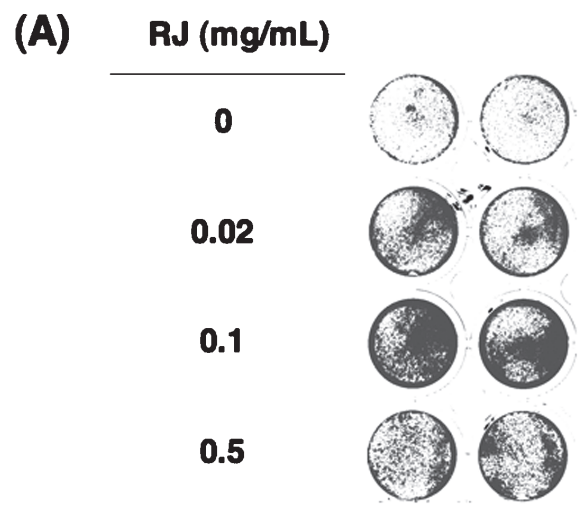

(B)

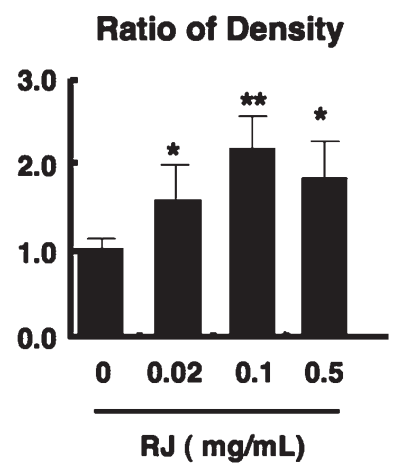

Fig. 3 Effect of RJ on mineralization of MPDL22 cells. (A) Effect of RJ $(0.02,0.1,0.5 \mathrm{mg} / \mathrm{mL})$ on mineralization in MPDL22 cells was examined by alizarin red staining after 12 days of culture in mineralization medium. Results show a representative experiment out of four identical assays. (B) The relative intensity of alizarin red staining was determined by image analysis. The data shown were quantified and normalized to those of alizarin red staining without RJ. RJ significantly enhanced mineralized nodule formation in MPDL22 cells. The values are the means \pm SD of four different experiments. ${ }^{*} P<0.05$ and ${ }^{\star \star} P<0.01$ compared with MPDL22 cells cultured with mineralization medium in the absence of RJ. 
(A)

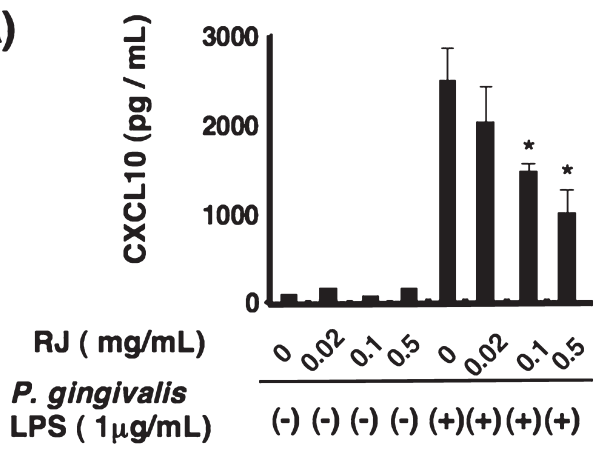

(B)

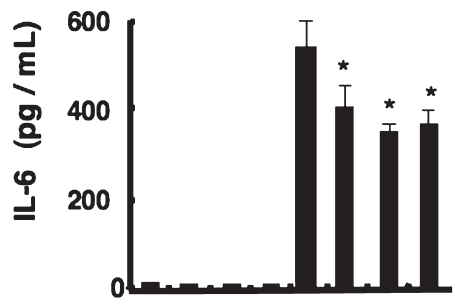

RJ ( $\mathrm{mg} / \mathrm{mL}) \quad 0$ ?

$P$. gingivalis
LPS $(1 \mu \mathrm{g} / \mathrm{mL})$$\quad(-)(-)(-)(-)(+)(+)(+)(+)$
(C)

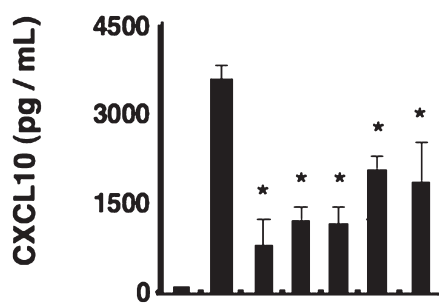

P. gingivalis LPS $(1 \mu \mathrm{g} / \mathrm{mL})$

\section{RJ ( $0.5 \mathrm{mg} / \mathrm{mL})$ \\ Pretreatment time ( hour)}

$(-)(+)(+)(+)(+)(+)(+)$

$(-)(-)(+)(+)(+)(+)(+)$

64210
(D)

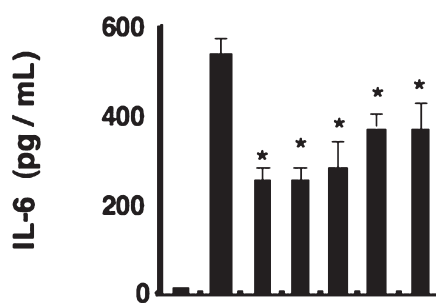

P. gingivalis
LPS $(1 \mu \mathrm{g} / \mathrm{mL})$

RJ ( $0.5 \mathrm{mg} / \mathrm{mL}$ )

Pretreatment time ( hour)

$$
(-)(+)(+)(+)(+)(+)(+)
$$

$(-)(-)(+)(+)(+)(+)(+)$

$\begin{array}{lllll}6 & 4 & 2 & 1 & 0\end{array}$

Fig. 4 Effect of RJ on CXCL10 and interleukin-6 (IL-6) production in MPDL22 cells. The effect of RJ on CXCL10 (A) and IL-6 (B) production in MPDL22 cells. MPDL22 cells were pretreated with RJ prior to LPS stimulation, and supernatants were collected after $24 \mathrm{~h}$. CXCL10 and IL-6 concentrations were determined by ELISA. The results represent the mean values \pm SD obtained from triplicate cultures. Next, MPDL22 cells were exposed to $0.5 \mathrm{mg} / \mathrm{mL}$ RJ either simultaneously, or up to $6 \mathrm{~h}$ before activation with LPS, and supernatants were collected after $24 \mathrm{~h}$. CXCL10 (C) and IL-6 (D) concentrations were determined by ELISA. The values represent the means \pm SD obtained from triplicate cultures. RJ suppressed CXCL10 and IL-6 production in MPDL22 cells. ${ }^{*} P<0.05$ compared with LPS-stimulated MPDL22 cells in the absence of RJ.

(A)

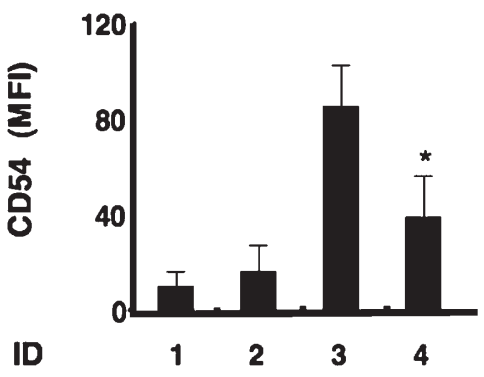

\section{RJ $(0.5 \mathrm{mg} / \mathrm{mL})$ \\ P. gingivalis}

(B)

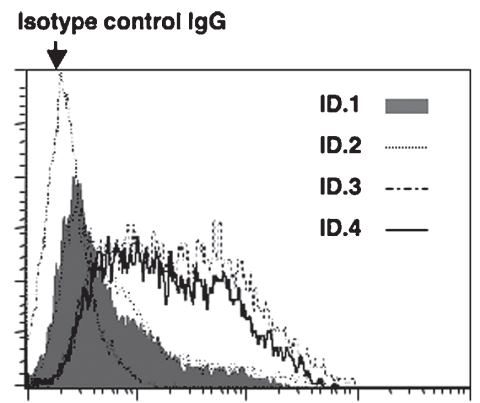

\section{CD54 expression}

Fig. 5 Effect of RJ on CD54 expression in MPDL22 cells. MPDL22 cells were treated with RJ (0.5 mg/mL) and/or LPS $(1 \mu \mathrm{g} / \mathrm{mL})$. MPDL22 cells were collected after $24 \mathrm{~h}$. The expression levels of CD54 in MPDL22 cells were determined by flow cytometry. (A) The results are expressed as mean fluorescent intensity (MFI). The data represent the mean \pm SD obtained from three different experiments. ${ }^{*} P<0.05$ compared with LPS-stimulated MPDL22 cells in the absence of RJ. (B) Fluorescence histogram of CD54 on surface of MPDL22 cells. RJ suppressed CD54 expression in MPDL22 cells stimulated with LPS. The istgramdata is representative of three different experiments. ID. 1: non-treated MPDL22 cells, ID. 2: RJ-treated MPDL22 cells, ID. 3: LPS-stimulated MPDL22 cells, ID. 4: LPS-stimulated MPDL22 cells after RJ-treatment. 
the estrogenic compounds in RJ should aid in the understanding of its involvement in bone metabolism.

$P$. gingivalis is a black-pigmented Gram-negative anaerobe and one of the causative microorganisms for the development of chronic inflammation in the periodontium (14). This is the first report that LPS of $P$. gingivalis can induce secretion of IL-6 and CXCL10 from mouse PDL cells. In human PDL cells, some reports have documented that stimulation by LPS derived from $P$. gingivalis (27) or Escherichia coli (23) induced IL-6, IL-8, IL-1 $\beta$ and tumor necrosis factor (TNF)- $\alpha$ production. In this study, we also revealed that RJ possesses inhibitory effects on LPS-stimulated IL-6 and CXCL10 production. IL-6 is a pleiotropic cytokine with a wide range of biological functions including immune responses and bone metabolism (1). CXCL10 is a chemoattractant for $\mathrm{T}$ helperl cells, which are suggested to be involved in alveolar bone destruction in periodontal diseases (9). A previous study reported that RJ suppressed IL- 6 and TNF- $\alpha$ production by LPS and interferon- $\gamma$ stimulated mouse macrophages (11), which is consistent with our observation.

CD54 is a cell surface adhesion molecule whose expression is increased at inflammatory sites such as in the periodontal tissues of periodontitis patients (6). Bacterial components or IL-1 $\beta$ have been reported to upregulate CD54 expression in human PDL cells $(8,12)$. Consistently, we found that LPS of $P$. gingivalis induced CD54 expression in MPDL22 cells. Furthermore, we revealed that RJ reduced CD54 expression in LPS-stimulated MPDL22 cells (Fig. 5). As anti-inflammatory compounds in RJ, we speculate that adenosine and adenosine derivatives, which have been identified as active components in RJ (5), could be candidates for suppressing CD54 expression in MPDL22 cells. Supporting this, our previous study showed that IL-1 $\beta$-induced CD54 expression in human gingival fibroblasts was abrogated by adenosine (16). Adenosine and/or other antiinflammatory compounds in RJ may contribute to the reduction of inflammatory responses in periodontal lesions.

In summary, our present results showed that RJ enhanced osteoblastic metabolism and exerted antiinflammatory effects. This work suggests that several constituents in RJ may be of benefit to oral and periodontal health. Elucidation of the mechanisms of the effects of RJ in mouse and human PDL cells requires further investigation.

\section{Acknowledgments}

This work was supported in part by Grants-in-Aid for Scientific Research (No. 20390529, 20390530, 20592427) from the Ministry of Education, Culture, Sports, Science and Technology, Japan. This work was also supported by Yamada Bee Farm Grant for Honeybee Research (to M.Y.). This funding source had no involvement in the study design, collection, analysis and interpretation of data, writing of the report, and the decision to submit the paper for publication. The other authors have declared that they have no conflict of interest.

\section{REFERENCES}

1. Akira S, Taga T and Kishimoto T (1993) Interleukin-6 in biology and medicine. Adv Immunol 54, 1-78.

2. Arceo N, Sauk J, Moehring J, Foster R and Somerman M (1991) Human periodontal cells initiate mineral-like nodules in vitro. J Periodontol 62, 499-503.

3. Bincoletto $C$, Eberlin S, Figueiredo C, Luengo $M$ and Queiroz M (2005) Effects produced by Royal Jelly on haematopoiesis: relation with host resistance against Ehrlich ascites tumour challenge. Int Immunopharmacol 5, 679-688.

4. Fujiwara S, Imai J, Fujiwara M, Yaeshima T, Kawashima T and Kobayashi K (1990) A potent antibacterial protein in royal jelly. Purification and determination of the primary structure of royalisin. J Biol Chem 265, 11333-11337.

5. Hattori N, Nomoto H, Mishima S, Inagaki S, Goto M, Sako $\mathrm{M}$ and Furukawa S (2006) Identification of AMP N1-oxide in royal jelly as a component neurotrophic toward cultured rat pheochromocytoma PC12 cells. Biosci Biotechnol Biochem 70, 897-906.

6. Hayashi J, Saito I, Ishikawa I and Miyasaka N (1994) Effects of cytokines and periodontopathic bacteria on the leukocyte function-associated antigen 1/intercellular adhesion molecule 1 pathway in gingival fibroblasts in adult periodontitis. Infect Immun 62, 5205-5212.

7. Hidaka S, Okamoto Y, Uchiyama S, Nakatsuma A, Hashimoto K, Ohnishi S and Yamaguchi M (2006) Royal jelly prevents osteoporosis in rats: beneficial effects in ovariectomy model and in bone tissue culture model. Evid Based Complement Alternat Med 3, 339-348.

8. Joe BH, Borke JL, Keskintepe M, Hanes PJ, Mailhot JM and Singh BB (2001) Interleukin-1beta regulation of adhesion molecules on human gingival and periodontal ligament fibroblasts. J Periodontol 72, 865-870.

9. Kawai T, Eisen-Lev R, Seki M, Eastcott JW, Wilson ME and Taubman MA (2000) Requirement of B7 costimulation for Th1-mediated inflammatory bone resorption in experimental periodontal disease. J Immunol 164, 2102-2109.

10. Kinane D (2001) Causation and pathogenesis of periodontal disease. Periodontol 2000 25, 8-20.

11. Kohno K, Okamoto I, Sano O, Arai N, Iwaki K, Ikeda M and Kurimoto M (2004) Royal jelly inhibits the production of proinflammatory cytokines by activated macrophages. Biosci Biotechnol Biochem 68, 138-145.

12. Lee S, Kim K and Choi B (2005) Upregulation of intercellular adhesion molecule 1 and proinflammatory cytokines by the major surface proteins of Treponema maltophilum and 
Treponema lecithinolyticum, the phylogenetic group IV oral spirochetes associated with periodontitis and endodontic infections. Infect Immun 73, 268-276.

13. Lerner U (2006) Inflammation-induced bone remodeling in periodontal disease and the influence of post-menopausal osteoporosis. J Dent Res 85, 596-607.

14. Mayrand D and Holt SC (1988) Biology of asaccharolytic black-pigmented Bacteroides species. Microbiol Rev 52, 134 152.

15. Mishima S, Suzuki K, Isohama Y, Kuratsu N, Araki Y, Inoue $\mathrm{M}$ and Miyata T (2005) Royal jelly has estrogenic effects in vitro and in vivo. $J$ Ethnopharmacol 101, 215-220.

16. Murakami S, Hashikawa T, Saho T, Takedachi M, Nozaki T, Shimabukuro Y and Okada H (2001) Adenosine regulates the IL-1 beta-induced cellular functions of human gingival fibroblasts. Int Immunol 13, 1533-1540.

17. Nagai T, Nagashima T, Myoda T and Inoue R (2004) Preparation and functional properties of extracts from bee bread. Nahrung 48, 226-229.

18. Nagatomo K, Komaki M, Sekiya I, Sakaguchi Y, Noguchi K, Oda S, Muneta T and Ishikawa I (2006) Stem cell properties of human periodontal ligament cells. J Periodontal Res 41, 303-310.

19. Narita Y, Nomura J, Ohta S, Inoh Y, Suzuki KM, Araki Y, Okada S, Matsumoto I, Isohama Y, Abe K, Miyata T and Mishima S (2006) Royal jelly stimulates bone formation: physiologic and nutrigenomic studies with mice and cell lines. Biosci Biotechnol Biochem 70, 2508-2514.

20. Okamoto I, Taniguchi Y, Kunikata T, Kohno K, Iwaki K, Ikeda M and Kurimoto M (2003) Major royal jelly protein 3 modulates immune responses in vitro and in vivo. Life Sci 73, 2029-2045.
21. Seo B, Miura M, Gronthos S, Bartold P, Batouli S, Brahim J, Young M, Robey P, Wang C and Shi S (2004) Investigation of multipotent postnatal stem cells from human periodontal ligament. Lancet 364, 149-155.

22. Shinoda M, Nakajin S, Oikawa T, Sato K, Kamogawa A and Akiyama Y (1978) [Biochemical studies on vasodilative factor in royal jelly (author's transl)]. Yakugaku Zasshi 98, 139145. (In Japanease)

23. Shu L, Guan SM, Fu SM, Guo T, Cao M and Ding Y (2008) Estrogen modulates cytokine expression in human periodontal ligament cells. $J$ Dent Res 87, 142-147.

24. Socransky S, Smith C and Haffajee A (2002) Subgingival microbial profiles in refractory periodontal disease. $J$ Clin Periodontol 29, 260-268.

25. Vittek J (1995) Effect of royal jelly on serum lipids in experimental animals and humans with atherosclerosis. Experientia 51, 927-935.

26. Yamada S, Tomoeda M, Ozawa Y, Yoneda S, Terashima Y, Ikezawa K, Ikegawa S, Saito M, Toyosawa S and Murakami S (2007) PLAP-1/asporin, a novel negative regulator of periodontal ligament mineralization. $J$ Biol Chem 282, 23070 23080

27. Yamaji Y, Kubota T, Sasaguri K, Sato S, Suzuki Y, Kumada $\mathrm{H}$ and Umemoto $\mathrm{T}$ (1995) Inflammatory cytokine gene expression in human periodontal ligament fibroblasts stimulated with bacterial lipopolysaccharides. Infect Immun 63, 35763581.

28. Yanagita M, Kojima Y, Kawahara T, Kajikawa T, Oohara H, Takedachi M, Yamada S and Murakami S (2010) Suppressive effects of nicotine on the cytodifferentiation of murine periodontal ligament cells. Oral Diseases 16, 812-817. 